\title{
NRP1 Activates NF-кB Signaling Pathway and Initiates Proliferation in Keratinocytes
}

Nikoletta Nagy ${ }^{1,2,3 *}$, Katalin Farkas ${ }^{2}$, Sarolta Bacsa ${ }^{1}$, Istvan Balazs Nemeth ${ }^{1}$, Zsuzsanna Bata-Csorgo ${ }^{1,2}$, Lajos Kemeny ${ }^{1,2}$ and Marta Szell,3

${ }^{1}$ Department of Dermatology and Allergology, University of Szeged, Szeged, Hungary

${ }^{2}$ Dermatological Research Group of the Hungarian Academy of Sciences, University of Szeged, Szeged, Hungary

${ }^{3}$ Department of Medical Genetics, University of Szeged, Szeged, Hungary

\begin{abstract}
Background: Neuropilin-1 (NRP1) is a membrane-bound co-receptor of vascular endothelial growth factor receptors (VEGFRs) and type-A plexins. These complexes mediate the effects of ligands such as vascular endothelial growth factor $165\left(\right.$ VEGF $\left._{165}\right)$ and semaphorin $3 \mathrm{~A}(\mathrm{SEMA}-3 \mathrm{~A})$.
\end{abstract}

Aim: Our aims were to elucidate whether the expression of NRP1 is related to the proliferation or differentiation programme of keratinocytes and to identify putative downstream signaling pathways of NRP1 in keratinocytes.

Materials and methods: Synchronized HaCaT cells and paraffin-embedded tissue samples of psoriasis were used to modell highly proliferative stages of keratinocytes. Real-time RT-PCR-based expression array was used to analyse downstream signaling pathways in keratinocytes.

Results: Synchronized HaCaT cells revealed that the peak expression of NRP1 occurred 24 hours before the cells reached their highest proliferative activity. Immunohistochemical staining of the highly proliferative tissue samples demonstrated that the high level of NRP1 protein expression partially overlapped with the expression of Ki67-positive cells. Real-time RT-PCR-based expression array identified the NF-KB pathway as one putative downstream signaling pathway of NRP1-mediated signaling in HaCaT cells. VEGF ${ }_{165}$ and SEMA-3A treatment of $\mathrm{HaCaT}$ cells stably transformed with a reporter construct for the assessment of NF-KB activation led to upregulation of the NF-KB activity.

Conclusion: Our results suggest that the NRP1 might be involved in the regulation of keratinocyte proliferation and might activate NF-KB pathway as a putative downstream signaling pathway in keratinocytes.

Keywords: Neuropilin-1; Vascular endothelial growth factor 165; Semaphorin 3A; NF-кB pathway; Synchronized HaCaT cells; Realtime RT-PCR-based expression array

\section{Introduction}

Neuropilin-1 (NRP1), a membrane-bound non-tyrosine kinase acts as a co-receptor for vascular endothelial growth factor receptor 2 (VEGFR-2) and type-A plexins mediating the effects of ligands such as vascular endothelial growth factor $165\left(\mathrm{VEGF}_{165}\right)$ and semaphorin $3 \mathrm{~A}$ (SEMA-3A) [1-3]. NRP1 has a key role in angiogenesis, axon guidance, embryonic development, tumor cell growth and immune response $[4,5]$. Recent studies reported that NRP1 is expressed on the surface of primary human keratinocytes and $\mathrm{HaCaT}$ cells and its expression is partially overlapped with the expression of VEGFRs [6,7]. The coexpression of NRP1 with VEGFR-2 increased the binding of VEGF ${ }_{165}$ 4-fold and enhanced $\mathrm{VEGF}_{165}$-induced chemotaxis and mitogenicity [8-10]. Regarding the function of NRP1 in the skin, Kurschat et al. [6] suggested that NRP1 is primarily a SEMA receptor and might be involved in wound healing. Another functional role of NRP1 was reported by Beck et al. [10] who demonstrated that the depletion of NRP1 in normal epidermis prevents skin tumor intitiation [8].

NRP1 is also involved in the modulation of a wide range of downstream signaling pathways, e.g. it influences the p53/caspase pathway to promote endothelial cell survival [11]. When NRP1 functions as the co-receptor of VEGFR-2, it activates phosphoinositide3-kinase (PI3K) and Akt in lung cancer cells [12]. NRP1 also promotes cirrhosis of the rodent and human liver by enhancing platelet derived growth factor (PDGF) and transforming growth factor $\beta$ (TGF- $\beta$ ) signaling in hepatic stellate cells [13]. There are currently no data in the literature on the possible downstream signaling mechanisms of NRP1 in keratinocytes.

In this study, we set out to elucidate whether the expression of NRP1 is related to the proliferation or differentiation programme of keratinocytes and we also investigated which downstream signaling pathways are modulated by NRP1 in HaCaT cells.

\section{Materials and Methods}

\section{Skin biopsy samples}

From the enrolled psoriatic $(n=3)$ and healthy individuals $(n=3)$, 4-mm punch biopsies were collected under local anesthesia from the proximal part of the thigh. From the psoriatic patients, two biopsies were taken, one from the involved and one from the uninvolved skin. All skin biopsies were placed into formalin (Sigma-Aldrich, St. Louis, $\mathrm{MO}$ ) and incubated overnight at $4^{\circ} \mathrm{C}$. After paraffin embedding, 4-mmthick sections were cut for immunohistochemistry.

${ }^{*}$ Corresponding author: Dr. Nikoletta Nagy, Department of Dermatology and Allergology, University of Szeged, 6 Koranyi fasor, H-6720 Szeged, Hungary, Tel: +36-62-545278; Fax: +36-62-545954; E-mail: nikoletta.nagy@gmail.com

Received June 05, 2013; Accepted June 20, 2013; Published June 22, 2013

Citation: Nagy N, Farkas K, Bacsa S, Nemeth IB, Bata-Csorgo Z, et al. (2013) NRP1 Activates NF-KB Signaling Pathway and Initiates Proliferation in Keratinocytes. Int J Genomic Med 1: 102. doi:10.4172/2332-0672.1000102

Copyright: ( 2013 Nagy N, et al. This is an open-access article distributed under the terms of the Creative Commons Attribution License, which permits unrestricted use, distribution, and reproduction in any medium, provided the original author and source are credited. 
The investigation was approved by the Internal Review Board. Written informed consent was obtained from all donors, and the study was conducted in accordance with the Principles of the Declaration of Helsinki.

\section{Synchronized HaCaT cells}

Human HaCaT keratinocytes, kindly provided by Dr N. E. Fusenig (Heidelberg, Germany), were cultured and synchronized as described in detail in a previous publication [14]. Briefly, HaCaT keratinocytes after reaching $100 \%$ confluence were cultured for 5 days in Dulbecco's modified Eagle's medium (DMEM) containing 10\% fetal bovine serum (FBS), and the medium was then replaced by serum-free medium, in which the cells were grown for 1 week. The synchronized cells were trypsinized and were seeded into $75-\mathrm{cm}^{2}$ culture flasks at a density of 5 $\times 10^{3}$ cells $/ \mathrm{cm}^{2}$ in high-glucose DMEM containing $10 \%$ FBS. Samples for real-time RT-PCR were collected at $0,12,24,36,48,72,96$ and 168 hours after the end of synchronization, using TRIzol reagent.

\section{Real-time RT-PCR}

Total RNA was isolated from cell cultures with TRIzol reagent, following the instructions of the manual. cDNA was generated with oligo(dT) and random hexamer primers from $1 \mu \mathrm{g}$ of RNA, using the iScriptTM cDNA Synthesis Kit of Bio-Rad Laboratories (Hercules, CA, USA). Real-time RT-PCR was performed and RNA expression data were normalized to the $18 \mathrm{~S}$ ribosomal RNA. The used primer sets and TaqMan probes were described in detail in a previous publication [15].

Human Signal Transduction PathwayFinder ${ }^{\mathrm{rm}} \mathrm{RT}^{2}$ Profiler $^{\mathrm{ma}}$ PCR Array (PAHS-014; SABiociences Corporation, Frederick, MD, USA) was used allowing the investigation of the expression of 84 key genes representing 18 different signal transduction pathways (http://www. sabiosciences.com/rt_pcr_product/HTML/PAHS-014A.html). Data processing was carried out with the online program (PCR Array Data Analysis online program of Sabiosciences www.sabiosciences.com) developed by the manufacturer.

\section{Immunohistochemistry}

After formalin fixation and paraffin embedding, 4-mm-thick sections were placed on silanized slides, dewaxed in xylene for $3 \times 5$ minutes, and rehydrated in decreasing concentrations of ethanol and methanol for $2 \times 3$ minutes and $2 \times 2$ minutes, respectively. Sections were blocked against endogenous peroxidase in a mixture of $90 \mathrm{ml}$ of methanol and $3 \mathrm{ml}$ of $\mathrm{H}_{2} \mathrm{O}_{2}$ for 5 minutes. Antigen retrieval was performed in an autoclave $\left(105^{\circ} \mathrm{C}\right)$ for 3 minutes in citrate buffer $(\mathrm{pH}=6)$. After nonspecific antigen blocking, anti-NRP1 mouse monoclonal IgG antibody (A12, sc-5307, Santa Cruz Biotechnology; Heidelberg, Germany) was used in a dilution of 1:50 for 30 minutes. To obtain more sensitive reactions, the HRP polymer (UltraOne visualization system; LabVision Corporation, Fremont, CA) was used for 30 minutes. Semiquantitative analysis was based on the intensity of the DAB.

\section{Statistical analysis}

The results obtained on synchronized $\mathrm{HaCaT}$ cells were assessed by variance analysis: one-way ANOVA for independent or correlated samples. For the real-time RT-PCR-based array data, the significances of the changes in gene expressions were evaluated with the unpaired Student t-test. The level of statistical significance was set at $<0.05$. Statistical analyses were carried out using VassarStats (http:// faculty.vassar.edu/lowry/VassarStats.html) and Superarray (www. abiosciences.com/pcr/arrayanalysis.php) websites.

\section{Results}

\section{The expression of NRP1 precedes the high proliferative state of $\mathrm{HaCaT}$ cells}

In order to delineate the function of NRP1 in HaCaT cells and to investigate whether its expression is related to the proliferation or the differentiation stages of these cells, we investigated the levels of expression of NRP1 in synchronized HaCaT cells.

In the synchronized HaCaT model, the changes in the NRP1 mRNA level were monitored for 1 week after the cells were released from cell quiescence (Figure 1). The real-time RT-PCR results revealed that the NRP1 mRNA expression level was increased significantly 12 hours after the cells were released from cell quiescence (an 80-fold increase relative to the 0 -hour level; one-way ANOVA test, $\mathrm{p}<0.0001$ ). The highest peak in its expression level was detected 36 hours after the cells were released from quiescence (a 100-fold increase as compared with the 0-hour level; one-way ANOVA test, $\mathrm{p}<0.00001$ ), and the NRP1 expression level subsequently started to decrease gradually. At the end of the 1-week experimental procedure, the NRP1 mRNA expression level had dropped to the starting level seen in the 0 -hour samples.

\section{In psoriasis NRP1 is expressed strongly in Ki67-positive keratinocytes}

Since on of $\mathrm{H} 2 \mathrm{O} 2$ for 5 minutes. experiments on synchronized $\mathrm{HaCaT}$ cells suggested that the expression of NRP1 is related to the high proliferative states of keratinocytes, our next aim was to investigate whether highly proliferative skin diseases are characterized by an elevated NRP1 expression.

The immunohistochemical staining demonstrated that NRP1 was expressed in the basal and suprabasal layers of the epidermis, and in fibroblasts, endothelial cells and inflammatory cells of the dermis in the control healthy skin (Figure 2a). The staining pattern was similar, but a higher intensity of staining was observed in the uninvolved psoriatic skin (Figure 2b). The most intensive NRP1 staining was detected in the involved psoriatic skin (Figure 2c). In line with our immunohistochemical data, we detected highly elevated NRP1 mRNA

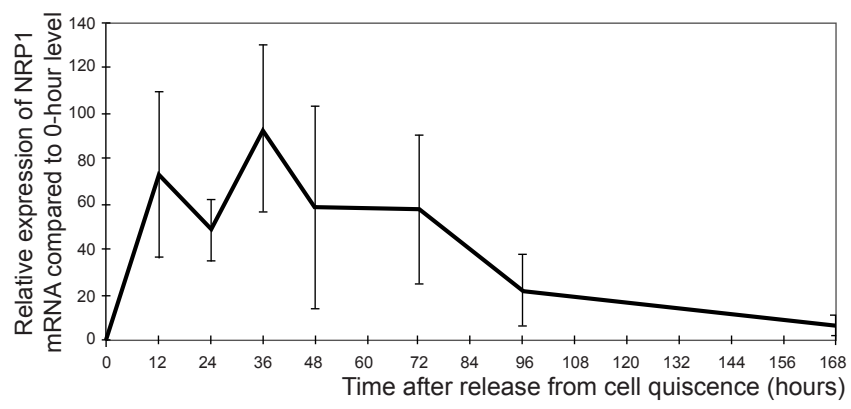

In the synchronized HaCaT model, changes in NRP1 mRNA levels were monitored by real-time RT-PCR for 1 week. 12 hours after cells were released from cell quiescence, 80-fold increase was measured in the expression of NRP1 mRNA (one-way ANOVA test, $p<0.0001$ ) relative to the amount measured at 0 hour in quiescent cells. The highest peak (a 100-fold increase) was detected 36 hours after cells were released from cell quiescence (one way ANOVA test, $p<0.00001)$. The NRP1 expression level then started to decrease gradually and dropped to the starting level seen in the 0-hour samples.

Figure 1: The highest relative expression of NRP1 mRNA was detected 36 hours after HaCaT cells were released from cell quiescence. 
Citation: Nagy N, Farkas K, Bacsa S, Nemeth IB, Bata-Csorgo Z, et al. (2013) NRP1 Activates NF-KB Signaling Pathway and Initiates Proliferation in Keratinocytes. Int J Genomic Med 1: 102. doi:10.4172/2332-0672.1000102

levels both in the epidermis (a 680 -fold increase; $n=3$ ) and in the dermis (a 75-fold increase; $n=3$ ) of psoriatic involved skin as compared with the healthy skin. We additionally examined the expression of Ki67, a well-established proliferation marker, in the same tissue samples (Figures 2d-2f). A comparison of the staining patterns of NRP1 and Ki67 revealed that the expression of NRP1 correlated well with the expression of Ki67. Not only proliferating Ki67 positive cells, but some of the Ki67 negative cells also showed positive staining for NRP1, this observation further support our hypothesis, that NRP1 is highly expressed in cells preceding proliferation and in proliferating cells.

\section{Real-time RT-PCR-based gene expression array identified the NF- $k B$ pathway}

In view of the broad ligand-binding ability of NRP1 and the fact that NRP1 may function as a co-receptor for several molecules, it is likely that NRP1 is involved in the modulation of a wide range of downstream signaling pathways [11-13]. There are as yet no literature data on the NRP1-activated downstream signaling mechanism in keratinocytes, and our aim was to identify which pathways are activated after treatment with VEGF $_{165}$ and SEMA-3A in HaCaT cells.

$\mathrm{HaCaT}$ cell cultures were grown to $80 \%$ confluence, and $20 \mathrm{ng} / \mathrm{ml}$ VEGF $_{165}$ or $10 \mathrm{ng} / \mathrm{ml} \mathrm{SEMA-3A}$ was added to the culture media. Six hours after $\mathrm{VEGF}_{165}$ or SEMA-3A treatment, the total RNA was isolated from all samples and the relative mRNA expression levels of 84 genes representing 18 signal pathways were measured by using real-time RTPCR-based arrays. Of the 84 investigated genes, 38 gave a detectable signal during the real-time RT-PCR measurements. Of these 38 genes, 16 displayed a relative change at least 4 -fold in its expression level due to VEGF $_{165}$, and 21 did so due to SEMA-3A treatment in comparison with the untreated control samples (Table 1). Both SEMA-3A and VEGF ${ }_{165}$ induced significant increases in the relative levels of expression of the TERT, IL-2, VEGFA and WNT2 genes suggesting activation of the NF$\mathrm{KB}$ pathway. $\mathrm{VEGF}_{165}$ treatment resulted in a marked upregulation of the BIRC3, IGFBP3 and TERT genes, suggesting activation of the p53, WNT and NF-кB pathways, while in SEMA-3A-treated keratinocytes we detected the upregulation of the BIRC3, VEGFA and TERT genes, also suggesting activation of the p53, WNT and NF- $\mathrm{KB}$ pathways. Both SEMA-3A and $\mathrm{VEGF}_{165}$ treatment resulted in a significant downregulation of the FAOXA2, FASLG and NRIP1 genes, pointing to inhibition of the Hedgehog, NFAT and Estrogen pathways.

\section{VEGF $_{165}$ and SEMA-3A treatment of NF-kB-HaCaT led to upregulation of NF- $\mathrm{KB}$}

Since our real-time RT-PCR-based gene array identified the NF$\kappa \mathrm{B}$ pathway as a putative downstream signaling pathway of NRP1 in $\mathrm{HaCaT}$ cells, we investigated the effects of $\mathrm{VEGF}_{165}$ and SEMA-3A in $\mathrm{HaCaT}$ cells stably transformed with an NF- $\kappa \mathrm{B}$-responsive element luciferase reporter gene construct (NF- $\kappa \mathrm{B}-\mathrm{HaCaT}$ ) [16]. Because TNFa is a potent enhancer of the NF- $\kappa B$ pathway, TNFa treatment was used as a positive control.

The NF- $\kappa \mathrm{B}-\mathrm{HaCaT}$ cultures $(\mathrm{n}=6)$ were treated with $50 \mathrm{ng} / \mathrm{ml}$ VEGF $_{165}, 10 \mathrm{ng} / \mathrm{ml}$ SEMA-3A or $10 \mathrm{ng} / \mathrm{ml}$ TNFa. Cells were collected 4 hours after the treatment and the activation of the NF- $\kappa B$ pathway was assessed by means of the luciferase assay. VEGF $_{165}$ and SEMA$3 \mathrm{~A}$ treatment resulted in a 2.9 -fold and a 1.9 -fold upregulation, respectively, while the positive control TNFa treatment led to a 4.8 -fold upregulation (Figure 3). The results obtained with NF- $\mathrm{kB}-\mathrm{HaCaT}$ are in line with the data on the real-time RT-PCR-based gene expression array, demonstrating induction of the NF- $\kappa \mathrm{B}$ pathway due to $\mathrm{VEGF}_{165}$ and SEMA-3A stimulation.

\section{Discussion}

Using a synchronized HaCaT keratinocyte model [14] (Figure 1), we demonstrated that the peak expression of NRP1 occurred 24 hours before the cells entered the S/G2/M phase and reached their highly proliferative states. These data allow the hypothesis that NRP1 is not
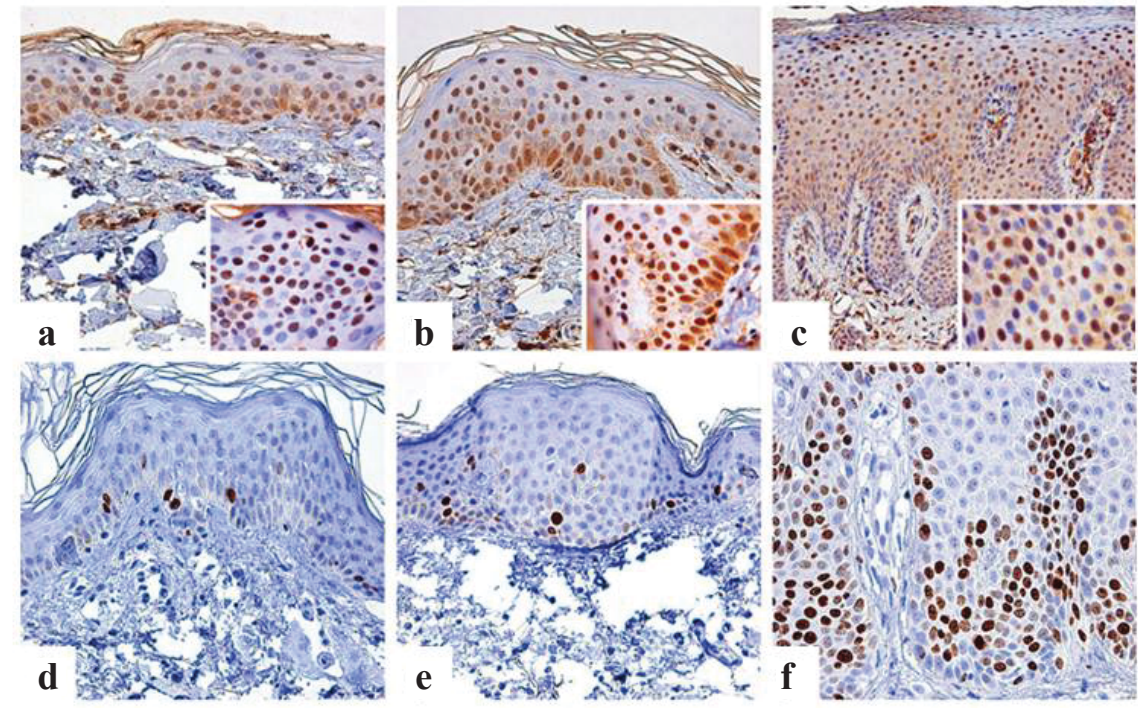

On immunohistochemistry, the NRP1 staining was positive in the basal and suprabasal layers of the epidermis, in the fibroblasts, endothelial cells and inflammatory cells of the dermis in the healthy skin (a). In the uninvolved psoriatic skin (b) increased, while in the uninvolved psoriatic skin (c) highly increased intensity of the NRP1 staining was detected compared to the staining of the healthy skin. The staining of NRP1 was partially overlapping with the staining of Ki67 in the healthy skin (d), in the uninvolved psoriatic skin (e) and in the uninvolved psoriatic skin (f).

Figure 2: NRP1 is expressed at a high level in hyperproliferative skin diseases. 
Citation: Nagy N, Farkas K, Bacsa S, Nemeth IB, Bata-Csorgo Z, et al. (2013) NRP1 Activates NF-kB Signaling Pathway and Initiates Proliferation in Keratinocytes. Int J Genomic Med 1: 102. doi:10.4172/2332-0672.1000102

Page 4 of 5

\begin{tabular}{|c|c|c|c|}
\hline Symbol & Fold change Co vs. VEGF ${ }_{165}$ & Fold change Co vs. SEMA-3A & Represented Pathways \\
\hline CCL20 & 0.06 & 0.38 & NF-kB \\
\hline IL1A & 1.06 & 0.55 & NF-kB \\
\hline IL2 & 2.96 & 14.42 & $N F-k B$ \\
\hline NAIP & 0.66 & 14.93 & NF-kB \\
\hline PECAM1 & 1.25 & 11.71 & $\mathrm{NF}-\mathrm{kB}$ \\
\hline TANK & 1.10 & 11.71 & $N F-k B$ \\
\hline TERT & 3.14 & 9.19 & NF-kB \\
\hline VEGFA & 2.96 & 14.42 & NF-kB \\
\hline WNT2 & 2.55 & 3.25 & $N F-k B$ \\
\hline BAX & 0.67 & 10.93 & p53 \\
\hline BIRC3 & 4.29 & 9.85 & p53 \\
\hline IGFBP3 & 1.18 & 0.55 & p53 \\
\hline MDM2 & 0.32 & 13.00 & p53 \\
\hline TP53 & 0.59 & 10.56 & p53 \\
\hline EGR1 & 0.10 & 0.32 & Phospholipase C \\
\hline ICAM1 & 0.15 & 0.42 & Phospholipase C \\
\hline NOS2A & 0.13 & 6.50 & Phospholipase C \\
\hline PTGS2 & 0.94 & 11.71 & Phospholipase C \\
\hline FAOXA2 & 0.12 & 0.28 & Hedgehog \\
\hline LTA & 2.93 & 3.86 & Hedgehog \\
\hline PTCH1 & 0.16 & 5.10 & Hedgehog \\
\hline WNT1 & 11.31 & 1.74 & Hedgehog \\
\hline IGFBP3 & 4.29 & 10.56 & WNT \\
\hline PPARG & 0.14 & 4.76 & WNT \\
\hline VEGFA & 0.68 & 9.85 & WNT \\
\hline BRCA1 & 0.11 & 2.30 & Estrogen \\
\hline NRIP1 & 0.16 & 0.14 & Estrogen \\
\hline EN1 & 0.65 & 0.54 & Retinoic acid \\
\hline RBP1 & 0.56 & 10.56 & Retinoic acid \\
\hline SELE & 2.68 & 2.07 & LDL \\
\hline VCAM1 & 2.60 & 2.38 & LDL \\
\hline KLK2 & 0.54 & 0.42 & Androgen \\
\hline TMEPAI & 0.16 & 0.66 & Androgen \\
\hline CYP19A1 & 1.11 & 16.56 & CREB \\
\hline ATF2 & 0.10 & 3.86 & Stress \\
\hline MMP7 & 0.16 & 0.50 & PI3-Akt \\
\hline CXCL9 & 1.57 & 17.15 & Jak-Stat \\
\hline FASLG & 0.12 & 0.18 & NFAT \\
\hline
\end{tabular}

Table 1: Pathways identified by real-time RT-PCR-based gene expression array analysis in $\mathrm{VEGF}_{165}$ and in SEMA-3A-stimulated HaCaT cells.

only associated with keratinocyte proliferation, but may be involved in proliferation initiation. Our data provide the first experimental evidence that the expression of NRP1 is related to keratinocyte proliferation.

Besides, the well-characterized model system of synchronized HaCaT keratinocytes, we studied NRP1 expression in psoriasis. Immunohistochemical stainings of NRP1 demonstrated an increased expression in psoriatic uninvolved skin, and a highly increased expression in psoriatic involved skin. The expression pattern of NRP1 partially overlapped with the Ki67 proliferation marker, lending further support to its association with cell proliferation.

However, the fact that the observed expression patterns of NRP1 and Ki67 were similar, but did not completely overlap in the examined tissue samples suggests that NRP1 might have other, as yet unknown functions in keratinocytes. Our in vitro expression data on NRP1 in synchronized $\mathrm{HaCaT}$ cells preceding proliferation indicate that it might be a protein contributing to the initiation of keratinocyte proliferation.

Accumulating evidence is suggestive of the enhancer role of
NRP1 on tumor growth and aggressiveness. NRP1 is known to be overexpressed in various human cancers, such as lung cancer, prostate cancer, pancreatic carcinoma and astrocytic tumors [12-17]. It has recently been reported that the expression of NRP1 is also upregulated in melanoma [16], moreover, the depletion of NRP1 in normal epidermis prevents skin tumor intitiation [8].

Real-time RT-PCR-based arrays demonstrated that stimulation of HaCaT keratinocytes with $\mathrm{VEGF}_{165}$ or SEMA-3A induced the activation of NF- $\kappa B$ pathway (Table 1). Besides NF- $\kappa B$, our data revealed more than 4-fold changes in the expression of other intracellular signaling molecules, indicating activation of the Hedgehog and p53 pathways and inhibition of the Phospholipase $\mathrm{C}$ pathway. To the best of our knowledge, this is the first report that describes the putative

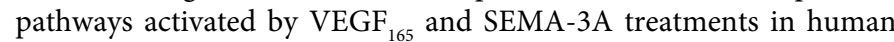
keratinocytes. At the same time, it must be noted that $\mathrm{VEGF}_{165}$ and SEMA-3A stimulations are not exclusively mediated by NRP1, since the effects of these ligands can also be mediated by other receptors, such as VEGFRs and type-A plexins [1-3].

In two independent experimental approaches (the gene expression 
Citation: Nagy N, Farkas K, Bacsa S, Nemeth IB, Bata-Csorgo Z, et al. (2013) NRP1 Activates NF-kB Signaling Pathway and Initiates Proliferation in Keratinocytes. Int J Genomic Med 1: 102. doi:10.4172/2332-0672.1000102

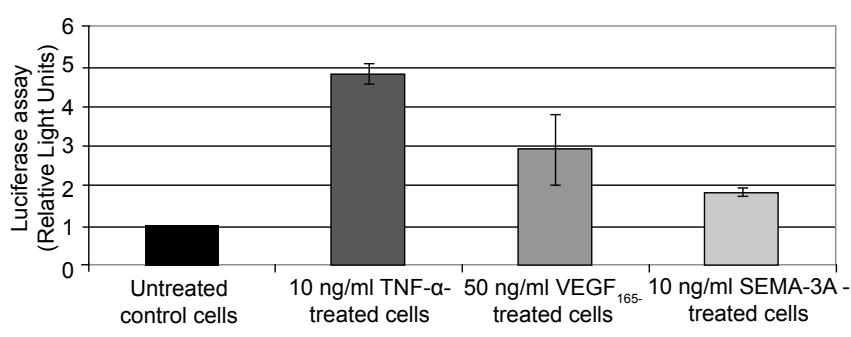

TNFa stimulation, a potent enhancer of the NF-KB pathway, was used as a positive control. The NF-kB-HaCaT cultures $(n=6)$ were treated with $50 \mathrm{ng} /$ $\mathrm{ml} \mathrm{VEGF}, 10 \mathrm{ng} / \mathrm{ml}$ SEMA-3A or $10 \mathrm{ng} / \mathrm{ml}$ TNFa. Cells were collected 4 hours after treatment and the activation of NF-KB pathway was assessed with luciferase assay. VEGF ${ }_{165}$ and SEMA-3A treatment resulted in a 2.9 -fold and a 1.9 fold upregulation, respectively, while the positive control TNFa treatment caused a 4.8-fold upregulation.

Figure 3: $\mathrm{VEGF}_{165}$ and SEMA-3A stimulation results in NF-KB activation of $\mathrm{HaCaT}$ cells stably transformed with an NF-kB responsive element - luciferase reporter gene construct (NF-kB-HaCaT).

array and use of the NF- $\kappa \mathrm{B}-$ luciferase reporter system in HaCaT cells), our results suggested that both $\mathrm{VEGF}_{165}$ and SEMA-3A activate NF$\kappa \mathrm{B}$ signaling in $\mathrm{HaCaT}$ cells, and the fact that the deleterious effects of NRP1 silencing in $\mathrm{HaCaT}$ cells could be reversed by $\mathrm{VEGF}_{165}$ treatment suggests that signaling of these molecules is mediated at least partially by NRP1 in keratinocytes, but other, as yet unidentified upstream signaling mechanism may also be involved.

In thi study we demonstrated that the expression of NRP1 is related to the high proliferative states of keratinocytes. We also report here the increased expression of NRP1 in psoriasis providing a putative new therapeutic target molecule for anti-psoriatic drug development. Moreover, the real-time RT-PCR-based gene expression array identified the NF- $\mathrm{kB}$ pathway as a putative downstream signaling mechanism for the ligands of NRP1, VEGF ${ }_{165}$ and SEMA-3A. Our data lead to the conclusion that the NRP1-VEGFR interaction exists in keratinocytes, since silencing of the NRP1 in keratinocytes caused severe morphologic changes and decreased proliferation, but these effects were rescued by VEGF $_{165}$ stimulation.

\section{Acknowledgments}

TÁMOP-4.2.1/B-09/1/KONV-2010-0005 grant

TÁMOP-4.2.2/B-10/1/KONV-2010-0012 grant

\section{Conflict of Interest}

The authors declare that they have no conflict of interest.

\section{References}

1. Shintani Y, Takashima S, Asano Y, Kato H, Liao Y, et al. (2006) Glycosaminoglycan modification of neuropilin-1 modulates VEGFR2 signaling. EMBO J 25: 3045-3055.

2. Hsieh SH, Ying NW, Wu MH, Chiang WF, Hsu CL, et al. (2008) Galectin-1, a novel ligand of neuropilin-1, activates VEGFR-2 signaling and modulates the migration of vascular endothelial cells. Oncogene 27: 3746-3753.

3. Sulpice E, Plouet J, Berge M, Allanic D, Tobelem G, et al. (2008) Neuropilin-1 and neuropilin-2 act as coreceptors, potentiating proangiogenic activity. Blood 111: 2036-2045.
4. Chen C, Li M, Chai H, Yang H, Fisher WE, et al. (2005) Roles of neuropilins in neuronal development, angiogenesis, and cancers. World J Surg 29: 271-275.

5. Kolodkin AL, Levengood DV, Rowe EG, Tai YT, Giger RJ et al. (1997) Neuropilin is a semaphorin III receptor. Cell 90: 753-762.

6. Kurschat P, Bielenberg D, Rossignol-Tallandier M, Stahl A, Klagsbrun M (2006) Neuron restrictive silencer factor NRSF/REST is a transcriptional repressor of neuropilin-1 and diminishes the ability of semaphorin $3 A$ to inhibit keratinocyte migration. J Biol Chem 281: 2721-2729.

7. Man XY, Yang XH, Cai SQ, Yao YG, Zheng M (2006) Immunolocalization and expression of vascular endothelial growth factor receptors (VEGFRs) and neuropilins (NRPs) on keratinocytes in human epidermis. Mol Med 12: 127136.

8. Soker S, Takashima S, Miao HQ, Neufeld G, Klagsbrun M (1998) Neuropilin-1 is expressed by endothelial and tumor cells as an isoform-specific receptor for vascular endothelial growth factor. Cell 92: 735-745.

9. Zachary I, Gliki G (2001) Signaling transduction mechanisms mediating biological actions of the vascular endothelial growth factor family. Cardiovasc Res 49: 568-581.

10. Beck B, Driessens G, Goossens S, Youssef KK, Kuchnio A, et al. (2011) A vascular niche and a VEGF-Nrpl loop regulate the initiation and stemness of skin tumours. Nature 478: 399-403.

11. Wang X, Zhang W, Cheever T, Schwarz V, Opperman K, et al. (2008) The C. elegans L1CAM homologue LAD-2 functions as a coreceptor in MAB-20/ Sema2 mediated axon guidance. J Cell Biol 180: 233-246.

12. Hong TM, Chen YL, Wu YY, Yuan A, Chao YC, et al. (2007) Targeting neuropilin 1 as an antitumor strategy in lung cancer. Clin Cancer Res 13: 4759-4768.

13. Cao Y, Szabolcs A, Dutta SK, Yaqoob U, Jagavelu K, et al.(2010) Neuropilin-1 mediates divergent R-Smad signaling and the myofibroblast phenotype. J Biol Chem 285: 31840-31848.

14. Pivarcsi A, Szell M, Kemeny L, Dobozy A, Bata-Csörgo Z (2001) Serum factors regulate the expression of the proliferation- related genes alpha5 integrin and keratin 1, but not keratin 10, in HaCaT keratinocytes. Arch Dermatol Res 293: 206-213.

15. Nagy N, Bata-Csorgo Z, Kopasz N, Szeg C, Pivarcsi A, et al. (2006) The expression of keratinocyte growth factor receptor (FGFR2-IIlb) correlates with the high proliferative rate of HaCaT keratinocytes. Exp Dermatol 15: 596-605.

16. Brychtova S, Bezdekova M, Brychta T, Tichy M (2008) The role of vascular endothelial growth factors and their receptors in malignant melanomas. Neoplasma 55: 273-279.

17. Parikh AA, Liu WB, Fan F, Stoeltzing O, Reinmuth N, et al. (2003) Expression and regulation of the novel vascular endothelial growth factor receptor neuropilin-1 by epidermal growth factor in human pancreatic carcinoma Cancer 98: 720-729. 